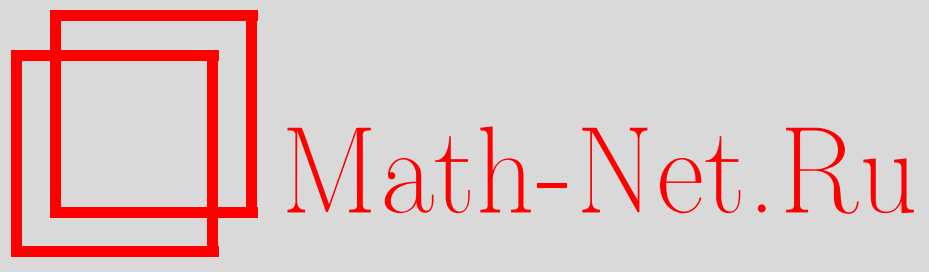

В. Х. Салихов, О мере иррациональности числа $\pi$, УМH, 2008, том 63, выпуск 3, 163-164

DOI: https://doi.org/10.4213/rm9175

Использование Общероссийского математического портала Math-Net.Ru подразумевает, что вы прочитали и согласны с пользовательским соглашением http: //www . mathnet.ru/rus/agreement

Параметры загрузки:

IP: 18.209 .158 .208

26 апреля 2023 г., $17: 29: 23$

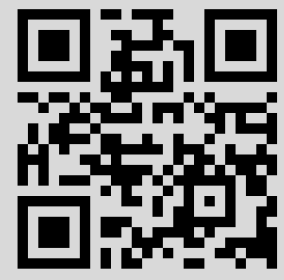




\section{О мере иррациональности числа $\pi$}

\section{В. Х. Салихов}

В 1953 г. К. Малер [1] получил первую оценку снизу порядка приближения числа $\pi$ рациональными дробями: $|\pi-p / q| \geqslant q^{-30}$ для всех $p, q \in \mathbb{N}, q \geqslant q_{0}$.

Позднее этот результат был улучшен: М. Миньотт [2] заменил показатель 30 на 20, а Г. Чудновский [3] - на $19.8899944 \ldots$. . В ряде работ М. Хата [4]-[6] снизил этот показатель до $8.016045 \ldots$. . Целью данной работы является доказательство следующего утверждения.

Теорема 1. Для всех $p, q \in \mathbb{N}, q \geqslant q_{0}$, выполняется неравенство

$$
\left|\pi-\frac{p}{q}\right| \geqslant q^{-\nu}, \quad \text { дде } \nu=7.6063 \ldots
$$

Улучшение результата М. Хата связано с рассмотрением интеграла:

$$
\begin{gathered}
J \equiv \int_{4-2 i}^{4+2 i} R(x) d x \\
R(x)=\frac{(x-4+2 i)^{3 n}(x-4-2 i)^{3 n}(x-5)^{3 n}(x-6+2 i)^{3 n}(x-6-2 i)^{3 n}}{x^{5 n+1}(10-x)^{5 n+1}},
\end{gathered}
$$

где $n$ - четно, $n \rightarrow+\infty$, путь интегрирования от $4-2 i$ до $4+2 i$ проходит через точки перевала $x_{1}$ и $x_{2}$ (которые мы укажем ниже) и точку $x_{3}=5$.

Перейдем к доказательству сформулированной теоремы. Очевидно, что $R(x) \in$ $\mathbb{Q}(x)$, и ввиду четности $n$ выполняется равенство $R(10-x)=R(x)$. Поэтому разложение $R(x)$ в сумму простейших дробей можно представить в следующем виде:

$$
R(x)=P(x)+\sum_{j=0}^{5 n}\left(\frac{a_{j}}{x^{j+1}}+\frac{a_{j}}{(10-x)^{j+1}}\right), \quad P(x)=\sum_{\nu=1}^{5 n-1} b_{\nu} x^{\nu-1},
$$

где все $a_{j} \in \mathbb{Q}$, все $b_{\nu} \in \mathbb{Z}$.

Имеем из (2) и (3)

$$
\varepsilon_{n}^{\prime} \equiv \frac{1}{i} J=\frac{1}{2} a_{0} \pi+r_{1}+r_{2}, \quad a_{0}, r_{1}, r_{2} \in \mathbb{Q} .
$$

Для вычисления асимптотики $\varepsilon_{n}^{\prime}$ и $a_{0}$ при $n \rightarrow \infty$ используется метод перевала, применяемый к интегралам $J$ и $a_{0}=\frac{1}{2 \pi i} \int_{|x|=1} R(x) d x$. Сначала вычислим знаменатель рациональных чисел $a_{0}, r_{1}$ и $r_{2}$.

Обозначим

$$
d(m)=\frac{(3 n) !}{(3 n-m) ! m !}, \quad m=0,1, \ldots, 3 n ; \quad d^{\prime}(m)=\frac{(5 n+m) !}{m !(5 n) !}, \quad m \in \mathbb{Z}^{+} .
$$

Пусть для $s=0,1, \ldots, 5 n$

$M_{s}=\left\{\bar{m}=\left(m_{1}, \ldots, m_{6}\right) \mid\right.$ все $\left.m_{r} \in \mathbb{Z}^{+} ; m_{r} \leqslant 3 n, r=1, \ldots, 5 ; m_{1}+\cdots+m_{6}=5 n-s\right\}$.

Пусть, наконец, $K=\{a+b i \mid a, b \in \mathbb{Z}\}-$ кольцо гауссовых чисел, и для $N \in \mathbb{Z}^{+}$ пусть $\theta_{N}=\{N / 2\}$ - дробная доля числа $N / 2$. Из (3) несложно получить представление вида

$$
\begin{gathered}
a_{j}=5^{-n-1+j} 2^{2.5 n-2+1.5 j-\theta_{j}} A_{j}, \quad A_{j} \in \mathbb{Z} \\
A_{j}=\sum_{\bar{m} \in M_{j}} \gamma(\bar{m}) \rho(\bar{m}), \quad \rho(\bar{m}) \in K, \quad \gamma(\bar{m})=d\left(m_{1}\right) \cdots d\left(m_{5}\right) d^{\prime}\left(m_{6}\right) .
\end{gathered}
$$

Следующая лемма является аналогом леммы 2.2 работы [6]. 
Лемма 1. Пусть $p$ - простое число, $T_{n}=\{p>\sqrt{5 n} \mid 1 / 3 \leqslant\{n / p\}<2 / 5\}$,

$$
\Lambda_{1}(n)=\prod_{p \leqslant \sqrt{5 n}} p^{[\log 5 n / \log p]}, \quad \Lambda_{2}(n)=\prod_{\sqrt{5 n}<p \leqslant 5 n} p, \quad \Lambda_{3}(n)=\prod_{p \in T_{n}} p,
$$

$\Delta_{n}=\Lambda_{1}(n) \Lambda_{2}(n) / \Lambda_{3}(n)$. Тогда для всех $j \in\{1, \ldots, 5 n\}, \bar{m} \in M_{j} \cup M_{j+1}$ справедливо $\left(\Delta_{n} \gamma(\bar{m})\right) / j \in \mathbb{N}$.

Применяя эту лемму и равенства (3), (5), получаем следующее утверждение.

Лемма 2. Пусть $Q_{n}=5^{n+1} 2^{-2.5 n+3} \Delta_{n}$. Тогда все $Q_{n} a_{0} / 2, Q_{n} r_{1}, Q_{n} r_{2} \in \mathbb{Z}$.

Имеем $\lim _{n \rightarrow \infty} n^{-1} \ln Q_{n}=5-(\psi(2 / 5)-\psi(1 / 3))+\ln 5-2.5 \ln 2 \equiv \chi, \quad \psi(x)=$ $\Gamma^{\prime}(x) / \Gamma(x)$.

Завершение доказательства оценки (1) проходит точно по схеме доказательства теоремы 1.2 из [6], поэтому ограничимся лишь некоторыми комментариями.

Обозначим (см. (2))

$$
\frac{(x-4+2 i)^{3}(x-4-2 i)^{3}(x-5)^{3}(x-6+2 i)^{3}(x-6-2 i)^{3}}{x^{5}(10-x)^{5}} \equiv g(t)=\frac{\left(t^{2}+6 t+25\right)^{3} t^{1.5}}{(25-t)^{5}},
$$

где $t=(x-5)^{2}$. Точки перевала функции $g(t)$ являются корнями уравнения

$$
\frac{1.5}{t}+\frac{3(2 t+6)}{t^{2}+6 t+25}-\frac{5}{t-25}=0, \quad \text { или } \quad t^{3}-76.2 t^{2}-305 t-375=0,
$$

и суть $t_{1,2}=-1.933883 \ldots \pm i 0.971403 \ldots, t_{3}=80.067767 \ldots$.

Участвующие в $(2)$ точки $x_{1,2}$ имеют вид $x_{1,2}=5+\sqrt{t_{1,2}}$, где $\operatorname{Re} \sqrt{t_{1,2}}<0$, т. е.

$$
x_{1}=5-0.339310 \ldots-i 1.431438 \ldots, \quad x_{2}=5-0.339310 \ldots+i 1.431438 \ldots .
$$

Применяя для линейной формы $\varepsilon_{n} \equiv Q_{n} \varepsilon_{n}^{\prime}$ (см. (4)) замечание 2.1 из [6], получим, что неравенство (1) выполняется для любого

$$
\nu>1-\frac{\chi+\ln \left|g\left(t_{3}\right)\right|}{\chi+\ln \left|g\left(t_{1}\right)\right|}=7.60630852 \ldots
$$

и теорема доказана.

Автор выражает благодарность В. В. Зудилину и Ю. В. Нестеренко за полезные замечания.

\section{Список литературы}

[1] K. Mahler, Nederl. Akad. Wetensch. Proc. Ser. A., 56 (1953), 30-42. [2] M. Mignotte, Bull. Soc. Math. France, 37 (1974), 121-132. [3] G. V. Chudnovsky, Lecture Notes in Math., 925, Springer, Berlin, 1982, 299-322. [4] M. Hata, J. Reine Angew. Math., 407 (1990), 99-125. [5] M. Hata, J. Number Theory, 43:1 (1993), 51-67. [6] M. Hata, Acta Arith., 63:4 (1993), $335-349$.

В. Х. Салихов (V.Kh. Salikhov)

Брянский государственный технический университет

E-mail: svdh@rambler.ru
Представлено А. В. Михалёвым Принято редколлегией 24.01.2008 